\title{
EFEITOS DO CONSERVADORISMO CONDICIONAL DECORRENTE DA CRISE ECONÔMICA SOBRE O INVESTIMENTO DAS COMPANHIAS ABERTAS BRASILEIRAS
}

\author{
EFFECTS OF CONDITIONAL CONSERVATISM DUE TO ECONOMIC CRISIS \\ ON THE INVESTIMENT OF BRAZILIAN PUBLIC FIRMS
}

\author{
JAILSON MANOEL SILVA DUARTE \\ Universidade Federal da Paraíba. Endereço: Campus I| Loteamento Cidade \\ Universitária | João Pessoa/PB | Brasil. \\ Dhttp://orcid.org/0000-0002-3443-3248 \\ jailsonduarte@hotmail.com
}

\section{LAURO VINICIO DE ALMEIDA LIMA}

Universidade Federal da Paraíba. Endereço: Campus I| Loteamento Cidade Universitária $\mid$ João Pessoa/PB $\mid$ Brasil.

(D) http://orcid.org/0000-0001-5474-5655

lvinicio2@gmail.com

\section{EDILSON PAULO}

Universidade Federal do Rio Grande do Sul. Endereço: Avenida Paulo Gama, n.110 | Farroupilha | Porto Alegre/RS | Brasil.

(D) http://orcid.org/0000-0003-4856-9039

e.paulo@uol.com.br

\section{RESUMO}

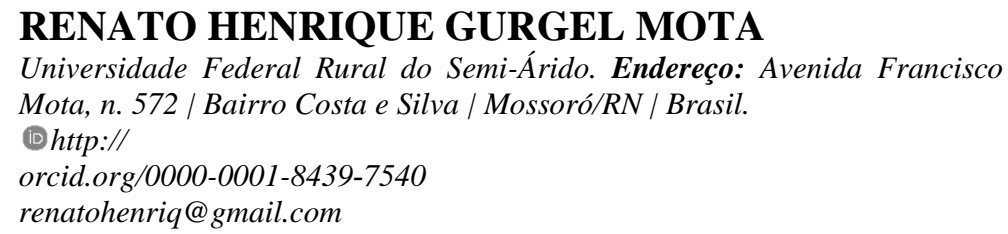

A pesquisa teve como objetivo identificar a influência do conservadorismo sobre o nível de investimento das companhias abertas em razão da crise econômica brasileira de 2014-2017. A pesquisa foi pautada em uma análise empírica por intermédio de coleta de informações relativas às empresas não financeiras de capital aberto, listadas no Brasil, Bolsa, Balcão $\left(\mathrm{B}^{3}\right)$, durante o período que compreendeu os exercícios de 2010 a 2018. Foi utilizado o modelo de Basu, ajustado para a mensuração do efeito da crise econômica brasileira no conservadorismo condicional das empresas. Em seguida, verificou-se a influência do nível de conservadorismo contábil destas, captado pelo modelo de Basu, nos investimentos em bens de capital das empresas. Os resultados indicam que, em períodos de crise econômica local, as empresas antecipam as futuras perdas aumentando o nível de conservadorismo. Esse comportamento conservador, entretanto, não foi capaz de mitigar os efeitos da crise, que influenciou negativamente o nível de investimentos de capital realizados pelas empresas brasileiras. Adicionalmente, observou-se que as empresas mais conservadoras reduzem significativamente seus investimentos em períodos de crise. Esses achados 
são relevantes para os investidores, pois estes podem considerar um comportamento conservador mais acentuado nos investimentos futuros das firmas. Cabe ressaltar também que os resultados da pesquisa podem auxiliar as políticas governamentais, no âmbito de que os gestores públicos busquem ações que viabilizem investimentos das empresas, minimizando os efeitos negativos da crise e permitindo um crescimento econômico mais rápido.

Palavras-chave: Crise Econômica Brasileira. Conservadorismo Contábil. Investimentos.

\begin{abstract}
This research aimed to identify the influence of accounting conservatism on the level of investments by traded companies due to the Brazilian economic crisis of 2014-2017. The research was based on an empirical analysis through the collection of information on non-financial public firms, listed in Brazil, Bolsa, Balcão $\left(B^{3}\right)$, during the period of 2010 to 2018. Basu model was adjusted to measure the effect of the Brazilian economic crisis on the conditional conservatism of firms. Then, using that model, the influence of the companies' accounting conservatism level on investments in Property, Plant, and Equipment $(P P \& E)$ was verified. The results indicate that in periods of local economic crisis, companies anticipate future losses by increasing the level of conservatism. This behavior, however, was not able to mitigate the effects of the crisis that negatively influenced the level of investments in PP\&E made by Brazilian companies. In addition, it has been observed that more conservative companies significantly reduce their investments in crisis times. These findings become relevant to investors as they may consider more conservative behavior in firms' future investments. It is also worth mentioning that the results of the research can help government policies in the sense that public managers seek actions that enable companies to invest, minimizing the negative effects of the crisis and allowing faster economic growth.
\end{abstract}

Keywords: Brazilian Economic Crisis. Accounting Conservatism. Investments.

\title{
1 INTRODUÇÃO
}

A crise do sistema financeiro norte-americano, ocorrida em 2008, desencadeou uma série de estudos na tentativa de compreender seu impacto sobre o nível de investimento das empresas. Esse período representou um inesperado choque negativo na oferta de financiamento externo, reduzindo significativamente a capacidade de as companhias realizarem novos investimentos (Duchin, Ozbas, \& Sensoy, 2010). Esse fato pode ser explicado também pelas empresas com restrições financeiras que, diante das dificuldades de acesso ao crédito, reduziram mais intensamente as despesas de capital nesse período (Campello, Graham, \& Harvey, 2010).

Segundo Duchin et al. (2010), as crises financeiras ou econômicas impactam diretamente o nível de investimento das firmas. Os autores evidenciaram que o investimento corporativo declina significativamente após o início da crise; ainda, o declínio é mais observado em empresas que apresentam as seguintes características: (a) baixa reserva de caixa; (b) alta dívida líquida de curto prazo; e (c) firmas com restrições financeiras ou que operam em setores dependentes de financiamento externo. Também, segundo Duchin et al. (2010), essa redução dos investimentos é fortemente explicada pela mudança nas oportunidades de investimento nesse período.

Pesquisas anteriores, como a de Biddle, Hilary e Verdi (2009), demonstraram que a qualidade das informações contábeis está relacionada à eficiência dos investimentos - ou seja, as empresas com maior qualidade dos relatórios financeiros são menos propensas a desviar do seu nível previsto de investimento. Os autores encontraram uma relação significativa entre a qualidade das informações contábeis e o nível de investimentos, dado que a redução da assimetria informacional pode mitigar os problemas de seleção adversa e do risco moral que dificultam a realização de investimentos de maneira eficiente. 
Uma das medidas de qualidade das informações contábeis é o conservadorismo contábil, descrito por Basu (1997) como a maior tendência da Contabilidade em reconhecer "notícias ruins" no resultado das firmas mais rapidamente do que "boas notícias" - por exemplo, as perdas não realizadas são normalmente reconhecidas mais cedo do que ganhos não realizados. Ainda segundo o autor, esse reconhecimento assimétrico entre boas e más notícias provoca diferenças sistemáticas na persistência dos lucros, prejudicando a previsibilidade de lucros futuros.

Essa relação entre o nível de conservadorismo contábil e os investimentos também foi estudada por Lara, Osma e Penalva (2016), que nela encontraram uma associação negativa - ou seja, as firmas mais conservadoras se apresentaram mais prudentes no desenvolvimento de projetos e na obtenção de dívidas quando inseridas em ambiente econômico mais desfavorável ao investimento, como período de crise econômica.

Sob a hipótese de que a crise econômica de 2008 foi um fator externo favorável para que as empresas reduzissem o nível de investimento de seus projetos, Balakrishnan, Watts e Zuo (2016) verificaram o efeito do conservadorismo contábil sobre o nível de investimento das empresas nesse período. Os autores encontraram evidências de que as empresas consideradas mais conservadoras sofreram menores reduções no nível de investimento de suas atividades quando comparadas com aquelas de menor comportamento conservador. Além disso, o efeito do conservadorismo sobre o investimento foi mais forte para as empresas que tinham dificuldades financeiras ou maiores necessidades de financiamento externo. Balakrishnan et al. (2016) partiram do pressuposto de que a classificação das empresas pelo seu nível de conservadorismo, ex ante o período de crise financeira no qual a empresa esteja inserida, ajuda a explicar a magnitude do declínio do investimento ex post.

Considerando que as informações das demonstrações financeiras das firmas são relevantes para os agentes do mercado de capitais e credores, pode-se esperar que em situações adversas, como períodos de crises econômicas, as empresas tendam a ser mais prudentes e não disponham de situações convenientes para investir mais intensamente em seus projetos.

Sendo assim, acredita-se que os efeitos dos indicadores macroeconômicos negativos, sinalizando para um período de crise econômica, são incorporados pela Contabilidade das empresas na tentativa de diluir os resultados negativos que seriam absorvidos em períodos futuros, por meio da antecipação do reconhecimento de futuras perdas. Sob essa percepção, acredita-se que o impacto das crises econômicas sobre o nível dos investimentos das empresas é menos acentuado naquelas que possuem números contábeis mais conservadores.

Nesse contexto, a economia brasileira passou por um período de forte desaquecimento (2014-2017), pois, desde 2014 o país apresenta diversos indicadores sinalizando um período de crise econômica, sendo que o Instituto de Pesquisa Econômica Aplicada (Ipea) (2016) estimou redução na atividade econômica do Brasil para os próximos anos e consequente retração dos investimentos por parte dos agentes econômicos, tendo em vista a redução no produto interno bruto (PIB) do país e as crescentes taxas de inflação.

A partir dessas considerações, acredita-se que os achados de Balakrishnan et al. (2016) são observáveis não somente quando surgem crises econômicas de dimensões globais, como em 2008, mas também em momentos de crise de natureza local, específica em um único país, como é o caso brasileiro. Diante do exposto, há o seguinte problema de pesquisa: Qual é o efeito do conservadorismo contábil no nível de investimentos em bens de capital nas companhias abertas brasileiras durante a crise econômica no Brasil entre os anos de 2014 e 2017?

Este trabalho objetiva analisar o efeito do conservadorismo contábil sobre o nível de investimento das empresas listadas no Brasil, Bolsa, Balcão $\left(\mathrm{B}^{3}\right)$, em razão da crise econômica brasileira entre aos anos de 2014 e 2017. Assim, foi avaliado se o grau de investimento de capital foi impactado na medida em que as empresas anteciparam o reconhecimento desses eventos em suas demonstrações contábeis - ou seja, se as empresas com maiores níveis de conservadorismo sofreram menores reduções nos investimentos do que as consideradas menos conservadoras. 
A realização deste estudo amplia as discussões levantadas por Balakrishnan et al. (2016) sobre a relação entre fatores macroeconômicos e informações contábeis divulgadas pelas firmas, em particular quanto ao seu nível de conservadorismo. Também busca revelar quais são os efeitos do conservadorismo contábil nos investimentos das empresas em momentos de crise econômica.

Portanto, a pesquisa contribui ao verificar, por meio de um estudo empírico, qual é o comportamento da relação entre o conservadorismo contábil e o nível de investimento das firmas em um período de crise econômica, considerando, especialmente neste estudo, uma análise em um mercado de capitais com características consideravelmente diferentes de estudos anteriores (Balakrishnan et al., 2016; Lara et al., 2016).

\section{REFERENCIAL TEÓRICO}

\subsection{Conservadorismo contábil}

Basu (1997) caracterizou o conservadorismo contábil como a tendência de os contadores exigirem um alto nível de verificação para reconhecer, nas demonstrações financeiras, as "boas notícias" mais do que as "más notícias" - isto é, os contadores preferem reconhecer mais tempestivamente as "notícias ruins" do que as "boas notícias". Consequentemente, o lucro é mais sensível em refletir publicamente, de forma oportuna, as "más notícias" disponíveis do que as "boas notícias".

Ball e Shivakumar (2005) segregam o conservadorismo em condicional e incondicional. Segundo os autores, em um ambiente de contratação, esse último parece, na melhor das hipóteses, neutro (sem viés) e possivelmente ineficiente (se o viés for desconhecido). Em contrapartida, o conservadorismo condicional envolve o reconhecimento oportuno de perdas. Coelho (2007) complementa essa diferenciação justificando que o conservadorismo incondicional reconhece, entre alternativas igualmente válidas, aquela que resulte em menor valor do patrimônio, e isto é válido tanto para receitas/ganhos quanto para despesas/perdas. O conservadorismo condicional privilegia o reconhecimento das más notícias, aquelas que reduzem o patrimônio, do que as boas.

No tocante à relação entre o conservadorismo contábil e o nível de investimento das empresas, Ahmed e Duellman (2011) analisaram o papel do conservadorismo contábil em relação ao monitoramento das decisões de investimento dos administradores no mercado acionário dos Estados Unidos, partindo do pressuposto de que o conservadorismo reduz os incentivos dos gestores ex ante de assumir projetos de valor presente líquido (VPL) negativo e ainda melhora o controle a posteriori dos investimentos. Assim, as empresas com resultados contábeis mais conservadores devem ter maior rentabilidade futura e menor susceptibilidade a mudanças na rubrica "itens especiais". Os resultados desse estudo indicaram que empresas mais conservadoras possuem margem bruta e fluxos de caixa futuros mais elevados e confirmaram a menor probabilidade de os itens especiais mudarem futuramente nas empresas consideradas menos conservadoras.

Bushman, Piotroski e Smith (2011) relacionaram diretamente o comportamento dos investimentos corporativos e o reconhecimento contábil oportuno das perdas econômicas em diferentes regimes contábeis. Os autores buscaram identificar a que ponto o reconhecimento de perdas pode influenciar as decisões de investimento por parte dos gestores, sob a perspectiva de que o comportamento dos investimentos é influenciado diante da deterioração de seu ambiente. $\mathrm{Na}$ pesquisa, os autores pressupõem que o reconhecimento das perdas terá impacto assimétrico sobre o comportamento dos investimentos, de modo que as decisões de investimento são influenciadas pelo declínio da oportunidade deste. Os resultados apontam que empresas conservadoras investem mais e emitem mais dívidas em ambientes propensos a falta de investimentos e que esses efeitos são mais observados em empresas caracterizadas por níveis 
maiores de assimetria informacional. Os autores também confirmam que o conservadorismo está associado à redução do investimento, até mesmo para aqueles em pesquisa e desenvolvimento.

Lara et al. (2016) defendem que o conservadorismo melhora a eficiência dos investimentos. Assim, acreditam que o conservadorismo resolve o conflito dívida-capital próprio, facilitando o acesso da firma ao financiamento de dívida e limitando subinvestimentos, isto é, projetos com VPL negativo, permitindo o financiamento de investimentos prudentes que não poderiam ser prosseguidos de outra forma.

Biddle e Hilary (2006) examinaram como a qualidade da informação contábil está relacionada ao nível de eficiência de investimento de capital das firmas. Os autores consideraram diversas medidas de qualidade contábil, derivadas de pesquisas anteriores e dois diferentes métodos para estimar a sensibilidade de fluxos de caixa de investimentos, sob a hipótese de que a qualidade dos números contábeis reforça a eficiência do investimento e reduz a assimetria informacional entre gestores e investidores. $\mathrm{O}$ estudo parte do pressuposto de que existe forte relação entre informação contábil e eficiência de investimento de capital em países com predominância de financiamento por capital próprio. Os resultados confirmam o argumento de que a Contabilidade é uma característica institucional disponível para as decisões políticas que aumentam a eficiência do investimento, mitigando a sensibilidade do fluxo de investimento, e que esse efeito é mais acentuado em situações nas quais o capital é fornecido por meio de transações independentes.

\subsection{A economia brasileira}

De acordo com Giambiagi (2011), desde os anos 1990, a economia brasileira passou por mudanças importantes, como o aumento do grau de abertura comercial e financeira, a maior competitividade das empresas, o aumento de privatizações e a maior atenção dada à inflação, além da adoção de medidas severas de ajuste fiscal. Ainda segundo o autor, tais medidas constituem etapas do processo de transformação de uma economia rumo a uma situação de maior competição com o exterior, e envolvem o objetivo de ter indicadores fiscais sólidos, inflação baixa e regras de política econômica relativamente estáveis.

Já nas primeiras décadas dos anos 2000, são registradas significativas controvérsias sobre o desempenho econômico brasileiro (Lopes, 2018). De um lado, destacam-se os argumentos que apontam para altas taxas de crescimento nesse período (Novy, 2009) e para a consolidação do processo de estabilização econômica e dos avanços sociais (Giambiagi, 2011). Por outro lado, ele também é visto como um período de redução da taxa média real de crescimento dos investimentos do Governo central e do crescimento real do PIB (Paulani, 2017). Seria, portanto, prematuro sustentar que o país passou por um processo de retomada do desenvolvimentismo e de uma nova fase econômica, apesar dos sinais visíveis da retomada de uma trajetória de crescimento e dos bons fundamentos macroeconômicos (Fonseca, Cunha, \& Bichara, 2013).

De acordo com o Instituto Brasileiro de Geografia e Estatística (IBGE) (2016), o PIB do país apresentou variação positiva no último trimestre de 2013 e terminou o ano com um aumento de 2,3\% em relação a 2012. Esse resultado positivo foi influenciado também pela taxa de investimento, que ficou ligeiramente acima do observado no ano anterior, chegando à marca de $18,4 \%$ do PIB.

Nesse período, os indicadores de inflação, embora não estivessem dentro das metas estabelecidas pelo Governo, apresentaram resultados toleráveis. O Índice Nacional de Preços ao Consumidor Amplo (IPCA) concluiu $2013 \mathrm{em} \mathrm{5,91 \% ,} \mathrm{um} \mathrm{pouco} \mathrm{maior} \mathrm{do} \mathrm{que} \mathrm{o} \mathrm{ano} \mathrm{anterior,}$ devido à variação mensal de $0,92 \%$, referente ao mês de dezembro em comparação a novembro daquele ano. Assim, em termos de inflação, a economia brasileira já apresentava sinais de variação negativa nos períodos seguintes.

Ao observar o IPCA no trimestre seguinte, verifica-se que esses sinais já começam a ficar mais acentuados. Assim, ao fim do primeiro trimestre de 2014, o IPCA apresentou variação de $2,18 \%$, acima da taxa de $1,94 \%$ do mesmo período de 2013 . Considerando os últimos doze meses, 
o índice foi para $6,15 \%$, ficando acima dos $5,68 \%$ relativos aos doze meses anteriores. Já o IPCA acumulado no ano de 2014 terminou em 6,41\%, acima dos 5,91\% do ano anterior.

Seguindo essa tendência de queda nos indicadores econômicos do país, o PIB de 2014 sofreu variação negativa de $0,2 \%$ no último trimestre do ano. Com isso, o PIB encerrou o ano de 2014 praticamente estável em relação a $2013(+0,1 \%)$. No entanto, para confirmar a tese de que o ano de 2015 foi marcado por um período de crise econômica no país, o valor do PIB fechou o último trimestre, na comparação com o mesmo período de 2014, com retração de 5,9\%, encerrando o ano com recuo de 3,8\% em relação a 2014 (IBGE, 2016).

Diante desses indicadores, percebe-se que houve alterações do nível de atividade econômica do país, conduzindo a conjuntura local a um período de recessão que se estendeu até o ano de 2017, quando o PIB brasileiro cresceu cerca de 1\%, após os anos de retração, mas sem recompor as perdas decorrentes da crise econômica, retornando ao patamar encontrado em 2011 (Barbosa Filho, 2017).

Diante da crise econômica instalada no Brasil durante 2014 e 2017, é possível que as empresas tenham retraindo seus investimentos em razão da insegurança quanto aos retornos econômicos do capital investido. Do mesmo modo, o conservadorismo contábil, ao tender a antecipar as notícias ruins, poderia suavizar os efeitos da crise nos investimentos em bens de capital das empresas. Assim, com base no estudo de Balakrishnan et al. (2016), nesse contexto de desaceleração da economia, acredita-se que as empresas que anteciparam em suas demonstrações contábeis essa tendência de retração, adotando uma postura mais conservadora, sentiram intensamente os impactos no nível de investimento de suas operações.

\section{PROCEDIMENTOS METODOLÓGICOS}

\subsection{Metodologia e amostra}

Esta pesquisa é um estudo empírico, de natureza descritiva, com as empresas participantes do mercado de capitais brasileiro. Para compor a amostra, foram escolhidas as empresas não financeiras que negociaram ações na $\mathrm{B}^{3}$ nos anos de 2010 a 2018. Os dados foram coletados na base de dados da Economatica ${ }^{\circledR}$ (2008). O estudo não analisou as empresas financeiras, tendo em vista que elas possuem estrutura de capital bastante específica e, ao serem comparadas com as demais, poderiam enviesar os resultados. Assim, inicialmente foram utilizadas 276 companhias. No entanto, foram excluídas aquelas cujas informações sobre preços de ações não estavam disponíveis - ou por não constar na base de dados ou por não terem realizado negociação na data referente à coleta dos dados. A Tabela 1 apresenta a distribuição da amostra por setor.

Tabela 1

\section{Distribuição da amostra por setor}

\begin{tabular}{lclc}
\hline \multicolumn{1}{c}{ Setor } & Quantidade & Setor & Quantidade \\
\hline Bens industriais & 40 & Saúde & 13 \\
Consumo cíclico & 65 & Tecnologia da informação & 5 \\
Consumo não cíclico & 18 & Telecomunicação & 4 \\
Materiais básicos & 24 & Utilidade pública & 39 \\
Petróleo, gás e bicombustíveis & 9 & & \\
\hline
\end{tabular}

TOTAL

217

Fonte: Elaborado pelos autores (2019).

A amostra desta pesquisa foi composta por nove setores, segundo a classificação da $\mathrm{B}^{3}$. Merecem destaque os setores de consumo cíclico, com 65 empresas, e bens industriais, com 40 . Ao todo, fizeram parte do estudo 217 empresas listadas no $\mathrm{B}^{3}$. 


\subsection{O efeito da crise econômica sobre o conservadorismo}

A mensuração do conservadorismo condicional das empresas foi realizada com base no modelo original proposto por Basu (1997). Depois, o modelo foi ajustado para verificar o efeito da crise econômica brasileira entre os anos de 2014 e 2017, utilizando os resultados apurados para testar a hipótese de pesquisa.

A equação do modelo original de Basu (1997) é descrita da seguinte forma:

$$
\frac{X_{i t}}{P_{i t-1}}=\alpha_{0}+\alpha_{1} D_{i t}+\alpha_{2} R_{i t}+\alpha_{3} R_{i t} D_{i t}+\varepsilon \quad \text { Equação (1) }
$$

em que:

$\mathrm{X}_{\mathrm{it}}$ é o lucro por ação das empresas $i$ no ano $t$;

$\mathrm{P}_{\mathrm{it}-1}$ é o preço por ação no início do ano;

$\mathrm{R}_{\mathrm{it}}$ é o retorno da empresa $i$ no ano $t$, pelo logaritmo de $\mathrm{P}_{\mathrm{it}} / \mathrm{P}_{\mathrm{it}-1}$;

$\mathrm{D}_{\mathrm{it}}$ é uma variável dummy que assume valor 1 quando $\mathrm{R}_{\mathrm{it}}<0$ e 0 para as demais situações.

A partir da Equação (1), desenvolveu-se o modelo econométrico utilizado nesta pesquisa para verificar a relação entre o conservadorismo condicional das empresas listadas na $\mathrm{B}^{3}$ e a crise econômica brasileira de 2014-2017. Portanto, o modelo utilizado pode ser descrito da seguinte maneira:

$$
\frac{\mathrm{X}_{\mathrm{it}}}{\mathrm{P}_{\mathrm{it}-1}}=\alpha_{0}+\alpha_{1} \mathrm{D}_{\mathrm{it}}+\alpha_{2} \mathrm{R}_{\mathrm{it}}+\alpha_{3} \mathrm{R}_{\mathrm{it}} \mathrm{D}_{\mathrm{it}}+\alpha_{4} \mathrm{CRISE}_{\mathrm{it}}+\alpha_{5} \mathrm{CRISE}_{\mathrm{it}} \mathrm{R}_{\mathrm{it}} \mathrm{D}_{\mathrm{it}}+\varepsilon \quad \text { Equação (2) }
$$

em que:

$\mathrm{X}_{\text {it }}$ é o lucro por ação das empresas $i$ no ano $t$;

$\mathrm{P}_{\text {it-1 }}$ é o preço médio da ação no mês de abril, pois, no Brasil, as informações contábeis do exercício anterior são apresentadas até 31 de março do ano subsequente, surtindo efeito no mercado mobiliário no mês de abril;

$\mathrm{R}_{\mathrm{it}}$ é o retorno da empresa $i$ no ano $t$, calculado pelo logaritmo de $\mathrm{P}_{\mathrm{it}} / \mathrm{P}_{\mathrm{it}-1}$;

$\mathrm{D}_{\text {it }}$ é uma variável dummy que assume valor 1 quando $\mathrm{R}_{\mathrm{it}}<0$ e $\mathrm{R}_{\mathrm{it}}<0$, e 0 para as demais situações;

CRISE é uma variável dummy à qual foi atribuído valor 1 para os anos entre 2014 e 2017, momento da crise econômica que o modelo se propõe a captar, e 0 para os demais anos.

Levando em consideração que o reconhecimento assimétrico das perdas econômicas indica que o lucro reflete as más notícias antecipadamente às boas notícias, a teoria subjacente ao modelo proposto é de que o preço das ações é mais importante que o lucro contábil porque reflete as informações recebidas de outras fontes além do lucro corrente (Basu, 1997; Kothari \& Sloan, 1992). Destarte, torna-se perceptível que o conservadorismo, no lucro contábil, será mais oportuno e sensível às más notícias do que às boas. Com isso, espera-se que os retornos negativos sejam mais significativos do que os positivos inesperados, pois o conservadorismo aumenta a oportunidade do reconhecimento do lucro na divulgação de más notícias (Ball \& Shivakumar, 2005; Basu, 1997).

No modelo proposto, o coeficiente $\alpha_{2}$ captura o efeito das boas e más notícias diante do lucro contábil. Entretanto, o reconhecimento assimétrico da perda econômica em relação aos ganhos irá representar o conservadorismo condicional, sendo este evidenciado nos coeficientes $\alpha_{1}$ e $\alpha_{3}$; espera-se que estes sejam significativos estatisticamente, e que a relação do primeiro seja negativa e a do segundo positiva. Do mesmo modo, espera-se que o coeficiente $\alpha_{5}$ apresente 
relação positiva e estatisticamente significativa, evidenciando o efeito da crise econômica brasileira, entre anos de 2014 e 2017, no conservadorismo incondicional das empresas no Brasil, caso ocorra aumento em seu nível em razão da crise.

\subsection{O efeito do conservadorismo sobre o nível de investimento}

Para investigar o efeito do conservadorismo contábil sobre o nível de investimento das empresas em períodos de crise econômica, adaptou-se a metodologia desenvolvida por Balakrishnan et al. (2016). Dessa maneira, adotou-se um modelo de regressão utilizando-se de uma estrutura comparativa do nível de investimento antes e durante o período de crise. Assim, ele examina se uma classificação de conservadorismo anterior à crise torna a empresa mais robusta no desenvolvimento de suas atividades, de modo a sofrer menos os efeitos da crise em seu nível de investimento.

O modelo econométrico empregado segue a seguinte especificação:

$$
\underset{\text { INVEST }_{i t}=\alpha_{i}+\beta_{1} \text { CRISE }_{i t}+\beta_{2} \operatorname{CONSV}_{i t-1}+\beta_{3} \text { CRISE }_{i t} \times \text { CONSV }_{i t-1}+\beta_{4} X_{i t}+\varepsilon_{i t}}{\text { Equação (3) }}
$$

em que:

INVEST $_{\text {it }}$ são o investimento e as despesas de capital incorridos por empresa $i$ no ano $t$ (com dados de 2010 a 2018), dividido pelo ativo total;

CRISE $_{\text {it }}$ é uma variável dummy à qual foi atribuído valor 1 para os anos entre 2014 e 2017 e 0 para o demais anos;

CONSV $V_{\mathrm{i}}$ é o conservadorismo contábil capturado pelo coeficiente $a_{3}$ da Equação (1) do modelo de Basu (1997), estimado por empresa entre 2010 e 2018;

$\mathrm{X}_{\mathrm{it}}$ é uma variável de controle - nesse caso, foi utilizado o Q de Tobin.

Seguindo o entendimento de Balakrishnan et al. (2016), $\beta_{1}$ captura o impacto da crise sobre o nível de investimento; espera-se um coeficiente negativo e significativo, indicando a existência de dificuldades para a execução dos investimentos. $\beta_{2}$ captura o efeito do conservadorismo contábil no investimento em bens de capital, e $\beta_{3}$ é o coeficiente de interesse que captura o efeito moderador do conservadorismo na crise econômica - para este, espera-se um valor significativamente positivo, sugerindo que o conservadorismo suavizaria o impacto negativo da crise sobre o nível de investimento. Com isso, estabelece-se esta hipótese:

H1: O conservadorismo contábil suaviza os efeitos negativos da crise econômica no investimento em bens de capital das empresas brasileiras.

Os dados utilizados na pesquisa foram trabalhados num conjunto de dados em painel, sendo apresentados em duas dimensões: corte transversal (cross-section) e séries temporais, referentes aos exercícios de 2010 a 2018. Nesse ponto, os dados em painel possibilitaram estudar as influências das variáveis explicativas sobre a variável dependente num conjunto de observações e ao longo do tempo (Greene, 2003).

Para a análise de dados em painel, foram realizados os testes de Chow, Hausman e Breusch-Pagan, para definição de qual é o melhor modelo de estimação para os dados da amostra - pooled, efeitos fixos ou efeitos aleatórios (Gujarati, 2006). Além destes, foram realizados os testes de Wooldridge e Wald modificado para dados em painel, a fim de observar a autocorrelação e a heterocedasticidade, respectivamente. 


\section{APRESENTAÇÃO E ANÁLISE DOS RESULTADOS}

\subsection{Estatística descritiva}

A Tabela 2 apresenta a estatística descritiva das variáveis de interesse e controle utilizadas na pesquisa e dizem respeito à amostra contendo, 217 empresas e totalizando 1734 observações.

Tabela 2

Estatística descritiva das variáveis utilizadas no modelo no período de 2010 a 2018

\begin{tabular}{ccccc}
\hline Variáveis & Média & Desvio padrão & Mínimo & Máximo \\
\hline X/P & $-0,6162$ & 2,8979 & $-21,2862$ & 1,3852 \\
R & $-0,0045$ & 0,1368 & $-3,0374$ & 6,3452 \\
INVEST & 0,0459 & 0,0994 & $-0,8438$ & 2,7899 \\
Q DE TOBIN & 1,3634 & 3,8709 & $-0,4038$ & 71,0238 \\
\hline
\end{tabular}

Nota. X/P é o lucro por ação dividido pelo preço médio da ação no período anterior; R é o retorno da empresa $i$ no ano $t$, calculado com logaritmo de $\mathrm{P}_{\mathrm{it}} / \mathrm{P}_{\mathrm{it}-1}$; INVEST $_{\mathrm{i}, \mathrm{t}}$ é o investimento de capital da empresa i no ano $t$ dividido pelo ativo total, $\mathrm{DR}_{\mathrm{it}}<0$.

Fonte: Elaborado pelos autores (2019).

O resultado do índice de divisão entre lucro por ação e preço médio da ação, na amostra, foi, em média, - 0,6162, enquanto a variável de retorno $(\mathrm{R})$ apresentou média negativa igual a 0,45\%; ou seja, as empresas utilizadas na amostra, em média, não apresentaram desempenho positivo ao mercado. Essa média indica possíveis influências de um contexto desfavorável ao desempenho do mercado de capitais, isto é, traz indícios de um momento de crise que se instalou no país. O investimento em bens de capital (INVEST) representou, na média, 4,59\% do ativo total das empresas para o período estudado.

\subsection{Conservadorismo e crise econômica}

Para a análise do conservadorismo no contexto da crise econômica de 2014 a 2017, foram utilizadas as demonstrações contábeis referentes aos exercícios financeiros de 2010 a 2018. Também se utilizou o preço médio das ações relativo ao último dia do mês de abril do respectivo exercício e seu retorno no exercício subsequente.

Desse modo, após os testes de pressupostos para estimação da regressão e relaxamento do pressuposto de normalidade dos dados com base no teorema do limite central, observou-se seu poder de explicação e a significância das variáveis, conforme a Tabela 3.

Tabela 3

Estimação da regressão para captar o efeito da crise sobre o conservadorismo

\begin{tabular}{|c|c|c|c|c|c|c|}
\hline & \multicolumn{3}{|c|}{ Equação (1) - Basu (1997) } & \multicolumn{3}{|c|}{ Equação (2) - Basu (1997) modificado } \\
\hline Invest & Coeficiente & p-valor & VIF & Coeficiente & p-valor & VIF \\
\hline $\mathrm{D}$ & $-0,7531$ & 0 & 1,03 & $-0,5328$ & 0 & 1,07 \\
\hline $\mathrm{R}$ & 0,1236 & 0,056 & 2,09 & 0,0307 & 0,575 & 2,1 \\
\hline $\mathrm{RD}$ & 5,8241 & 0,001 & 2,08 & 4,1637 & 0,004 & 2,23 \\
\hline CRISE & - & - & - & $-0,5774$ & 0 & 1,06 \\
\hline CRISERD & - & - & - & 18,0263 & 0,032 & 1,16 \\
\hline Intercepto & $-0,1979$ & 0,001 & - & 0,0323 & 0,514 & - \\
\hline$R^{2}$ ajustado & \multicolumn{3}{|c|}{0,0647} & \multicolumn{3}{|c|}{0,1189} \\
\hline \multicolumn{4}{|c|}{ Observações: 1734} & \multicolumn{3}{|c|}{ Empresas: 217} \\
\hline
\end{tabular}

Nota. $\frac{X_{i t}}{P_{i t-1}}=\alpha_{0}+\alpha_{1} D_{i t}+\alpha_{2} R_{i t}+\alpha_{3} R_{i t} D_{i t}+\alpha_{4} C_{R I S E}+\alpha_{5} C_{i t} I S E_{i t} R_{i t} D_{i t}+\varepsilon$, em que X/P é o lucro por ação dividido pelo preço médio da ação; R é o retorno da empresa $i$ no ano $t$, calculado com logaritmo de $\mathrm{P}_{\mathrm{i} t} / \mathrm{P}_{\mathrm{it}-1}, \mathrm{P}_{\mathrm{it}}-\mathrm{P}_{\mathrm{it}-1}$; $\mathrm{D}$ é uma variável dummy que assume valor 1 quando $\mathrm{R}_{\text {it }}<0$ e 0 para as demais situações; CRISE é uma variável dummy para os exercícios, sendo 1 para os anos de 2014 a 2017 , momento da crise econômica que o modelo se propõe a captar, e 0 para os demais anos; CRISERD é a variável CRISE multiplicada pela variável R e pela dummy D; e IC (95\%) é o intervalo de confiança a $95 \%$.

Fonte: Elaborado pelos autores (2019). 
Analisando a Tabela 3, na Equação 1 e 2, verifica-se que os resultados se coadunam à teoria subjacente, consoante Basu (1997), sendo que os coeficientes das variáveis D e RD são negativo e positivo, respectivamente, e significantes estatisticamente, sugerindo que as más notícias foram divulgadas mais oportunamente pela Contabilidade do que as boas notícias. Na Equação 2, quanto à variável CRISERD (interação da crise com o retorno), ela foi significativa a nível confiança de 5\%. Esses resultados indicam que a crise econômica brasileira de 2014-2017 $\left(\alpha_{5}=18,0263\right)$ influenciou o conservadorismo contábil condicional das firmas da amostra, aumentando seu nível quando comparada com períodos sem crise $\left(\alpha_{4}=4,1637\right)$.

Os resultados encontrados no mercado brasileiro estão em consonância com a literatura internacional (Al-Hroot, Al-Qudah, \& Alkharabsha, 2017; Balakrishnan et al., 2016), confirmando a hipótese da influência da crise no nível de conservadorismo das empresas listadas na $\mathrm{B}^{3}$. Sendo assim, passa-se a analisar o efeito desse período de crise sobre o nível de investimento das empresas, considerando as mais conservadoras.

Assim, foram estimadas as regressões da Equação (1) e (2), por empresa, para obtenção dos coeficientes, algo representado por $\alpha_{3}$ na Equação 1, para o conservadorismo contábil, e $\alpha_{5}$ na Equação 2, para o conservadorismo contábil em decorrência da crise. Os coeficientes estimados foram utilizados na Equação 3.

\subsection{Conservadorismo e investimento de capital}

Para a análise do efeito do conservadorismo contábil no investimento em bens de capital pelas empresas brasileiras, buscou-se o modelo mais adequado para o estudo; observaram-se as seguintes alternativas: modelo pooled (restrito), modelo de efeitos fixos (irrestrito) e modelo de efeitos aleatórios. Após aplicados os testes de Chow, Hausman e Breusch-Pagan, foi verificado que o modelo de efeitos aleatórios é o mais adequado para a análise dos dados.

No tocante à análise das correlações entre as variáveis nos dados em painel, aplicou-se o teste de Wooldridge, rejeitando a hipótese nula de não existência de autocorrelação (Prob > F = 0,0005). Quanto a análise de homocedasticidade dos dados, foi aplicado o teste de Wald modificado, rejeitando o $\mathrm{H}_{\mathrm{o}}$ de variação constante $\left(\right.$ Prob $>\mathrm{chi}^{2}=0$ ) e detectando-se, portanto, um comportamento heterocedástico. Diante disso, foi necessário estimar a regressão de forma robusta para a correção do problema.

Ultrapassados os testes de pressupostos do modelo regressivo, ao estimar a regressão descrita na Equação 3, foram estabelecidas regressões utilizando os dados em painel balanceado e desbalanceado, verificando-se que, nas duas formas, os resultados não foram alterados e não houve diminuição na eficiência do estimador. Assim, a Tabela 4 demonstra os resultados da regressão, com base no modelo adotado por Balakrishnan et al. (2016), em um painel desbalanceado para permitir a ampliação da quantidade de observações do estudo.

Tabela 4

Modelo de regressão entre investimento e conservadorismo contábil

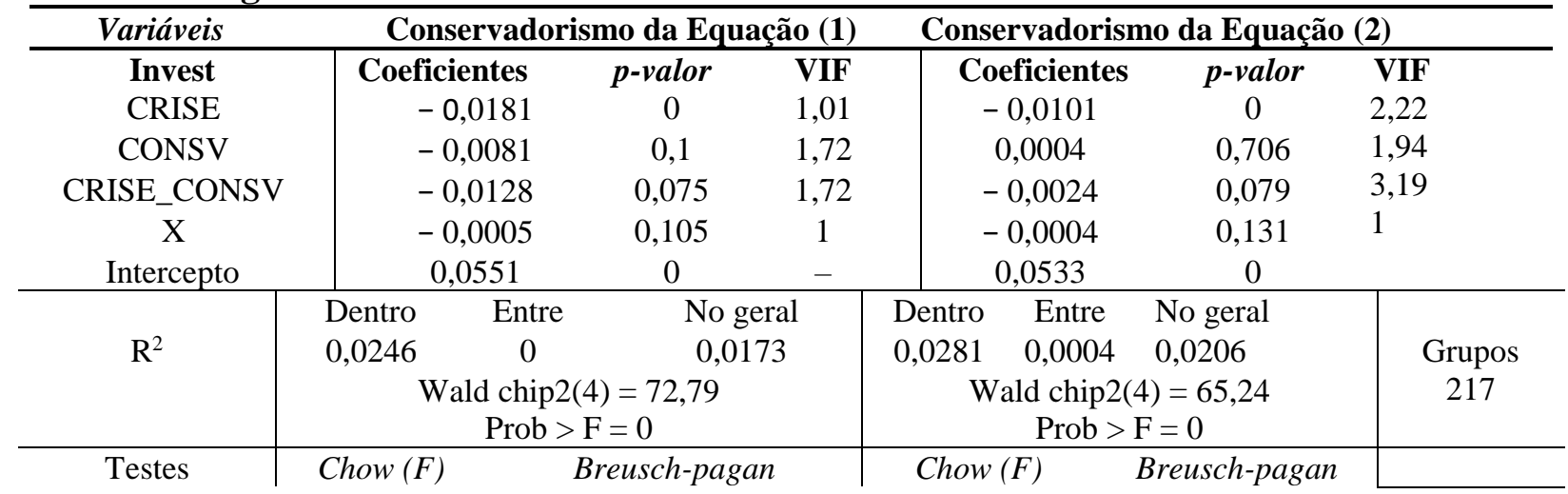



o investimento das companhias abertas brasileiras

\begin{tabular}{c|cc|ccc}
\hline & 0 & 0 & 0 & 0 & Obs. \\
Hausman & Wooldrigde & Hausman & Wooldrigde & 1734 \\
0,6537 & Wald & 0,0005 & 0,6306 & 0,0005 & \\
& 0 & \multicolumn{2}{c}{ Wald } \\
& & \multicolumn{2}{c}{0} & \\
\hline
\end{tabular}

Resultado dos testes: modelo de efeitos aleatórios

Nota. INVEST $_{i, t}=\alpha_{i}+\beta_{1}$ CRISE $_{i, t}+\beta_{2}$ CRISE $_{i, t} \times$ CONSV $_{i}+\beta_{3} X_{i, t}+\varepsilon_{i, t}$ INVEST $_{i, t}$ é o investimento de capital da empresa $i$ no ano $t$, dividido pelo ativo total; CRISE $_{\mathrm{i}, \mathrm{t}}$ é uma variável dummy para os exercícios, sendo 1 para os anos de 2014 a 2017, momento da crise econômica que o modelo se propõe a captar, e 0 para os demais anos; CONSV $_{\mathrm{i}}$ é a medida de conservadorismo conforme o modelo de Basu (1997); $\mathrm{X}_{\mathrm{i}, \mathrm{t}}$ é uma variável de controle Q de Tobin.

Fonte: Elaborado pelos autores (2019).

Conforme pode ser observado na Tabela 4, a suposição de que o sinal do coeficiente referente à crise seria negativo foi confirmada pelos dados. Assim, seguindo as proposições definidas por Balakrishnan et al. (2016), a variável CRISE com coeficiente negativo implica redução na capacidade de obtenção de financiamento, de modo a restringir o nível de investimento das empresas no momento de crise. No mercado brasileiro, a variável CRISE influenciou negativamente os investimentos em bens de capital em 1,81\%, em média, no período amostra.

O conservadorismo contábil (CONSV) captado pela Equação (1), por sua vez, apresentou relação negativa $(-0,0081)$ e significativa ao nível de $10 \%$. Esse resultado indica que, em média, empresas mais conservadoras reduzem seus investimentos em bens de capital, corroborando os achados de Lara et al. (2016), que chegaram à conclusão de que as firmas mais conservadoras são mais prudentes no desenvolvimento de projetos em períodos de crise econômica.

Ainda utilizando o conservadorismo contábil captado pela Equação (1), a interação entre conservadorismo e crise econômica apresentou coeficiente negativo (-0,0128), com significância estatística de 5\% - ou seja, o conservadorismo contábil moderado pela crise econômica exerceu influência negativa maior que o conservadorismo contábil em outros períodos. Esse resultado está em dissonância com a hipótese formulada na pesquisa e os resultados encontrados por Balakrishnan et al. (2016). Assim, ao contrário do esperado, há um indicativo de que, no período de crise no Brasil, o conservadorismo contábil não mitigou seu impacto negativo sobre os investimentos. Com isso, os resultados indicam que até mesmo as empresas participantes do mercado de capitais brasileiro que apresentaram conservadorismo contábil em maior grau não conseguiram manter seus investimentos em bens de capitais no momento em que o país enfrentava um processo de crise econômica.

Outrossim, utilizando o conservadorismo ajustado pelo momento de crise, captado pela Equação (2), verifica-se que a interação entre o conservadorismo e a crise econômica apresentou significância estatística, ao nível de $10 \%$, negativa no investimento (- 0,0024). O CONSV captado pela Equação (2) no momento de crise, unicamente considerado, não apresentou relação com significância estatística, sendo esse o único parâmetro divergente significativamente em relação às estimações do primeiro modelo.

Há que se destacar também que a variável de controle usada, Q de Tobin, nos dois modelos considerados, não apresentou significância estatística e econômica nos resultados das regressões. Destaque-se que há controvérsias sobre a relevância dessa métrica no Brasil no processo de tomada de decisão em investimentos de capitais (Santos, Costa, Alberto, Gonçalves, \& de Faria, 2011), de forma que, muitas vezes, é necessário estimá-la fazendo uso de adaptações (Kammler \& Wickstrom, 2009).

Diante dos resultados encontrados, pode-se verificar que também em períodos de crise econômica local, cujas proporções não são de natureza global, o comportamento conservador dos gestores exerceu influência no efeito da crise sobre o nível de investimento das empresas brasileiras - entretanto, em sentido contrário ao encontrado no mercado norte-americano. Ou seja, no Brasil, empresas com comportamento mais conservador, que apresentam maior 
conservadorismo contábil, reduziram seus investimentos diante da crise econômica entre o período de 2014-2017.

\section{CONSIDERAÇÕES FINAIS}

Conforme apresentado no problema de pesquisa, buscou-se analisar o efeito do conservadorismo contábil sobre o nível de investimento de bens das empresas listadas na $\mathrm{B}^{3}$ durante a crise econômica brasileira de 2014 a 2017. Com isso, o objetivo foi avaliar se o grau de investimento de capital foi impactado na medida em que as empresas anteciparam o reconhecimento desses eventos em suas demonstrações contábeis. Consequentemente, buscou-se verificar qual foi o efeito do nível de conservadorismo contábil das empresas nos investimentos em bens de capital.

A partir de uma abordagem empírica desenvolvida em duas etapas, a pesquisa revelou inicialmente significância estatística para o conservadorismo diante da crise econômica que perdurou de 2014 a 2017. Assim, os resultados indicam que uma contabilidade conservadora tende a inserir em suas demonstrações os possíveis resultados negativos que surgiriam em um futuro próximo. Logo, verificou-se que, de fato, as empresas brasileiras apresentam tendência de maior reconhecimento antecipado de más notícias durante a crise.

Outro achado do trabalho foi de que a crise de 2014-2017 afetou significativamente o nível de investimentos de capital das empresas brasileiras. Esse resultado já era esperado, pois em momentos de crise ocorre retração na oferta de recursos no mercado financeiro, afetando negativamente a captação das empresas para aplicar em novos investimentos.

Por fim, como propósito principal desta pesquisa, buscou-se verificar se empresas conservadoras são menos suscetíveis a redução no investimento no momento de crise. Os resultados não confirmaram essa hipótese, de forma que houve relação negativa significante entre conservadorismo e investimentos em momentos de crise. Desse modo, pode-se observar indícios de que as empresas, mesmo antecipando as futuras perdas em suas demonstrações contábeis, sofreram os impactos das crises econômicas no Brasil sobre seus investimentos.

Por todo o exposto, a pesquisa contribui com a discussão sobre o conservadorismo contábil, apresentando indícios de que, em períodos de crise, as empresas tendem a ser mais conservadores e já inserem nas suas demonstrações as perdas futuras diluídas ao longo dos períodos. Entretanto, no Brasil, as empresas persistiram com o impacto da crise nos investimentos em bens de capital, ainda que o conservadorismo contábil tenha, antecipadamente, assimilado as perdas.

Esses achados se tornam relevantes para os investidores, pois eles podem considerar um comportamento conservador mais acentuado nos investimentos futuros das firmas. Cabe ressaltar também que os resultados da pesquisa podem auxiliar as políticas governamentais, de maneira que os gestores públicos busquem ações que viabilizem os investimentos das empresas, minimizando os efeitos negativos da crise e permitindo um crescimento econômico mais rápido.

Em que pesem os resultados alcançados, reconhece-se as limitações impostas a este estudo, tendo em vista a utilização de apenas um modelo de conservadorismo. Sabe-se que outros modelos poderiam ser utilizados para tornar os resultados mais robustos, e outras variáveis de controle poderiam ser utilizadas para verificar melhor o impacto sobre os investimentos. Portanto, recomenda-se que novas pesquisas sejam realizadas nessa temática, ampliando o escopo desta pesquisa.

\section{REFERÊNCIAS}

Al-Hroot, Y. A., Al-Qudah, L. A. M., \& Alkharabsha, F. I. A. (2017). The effect of the global financial crisis on the level of accounting conservatism in commercial banks: Evidence from Jordan. International Journal of Business and Management, 12(2), 151-159. 
Ahmed, A. S., \& Duellman, S. (2011). Evidence on the role of accounting conservatism in monitoring managers' investment decisions. Accounting \& Finance, 51(3), 609-633.

Balakrishnan, K., Watts, R., \& Zuo, L. (2016). The effect of accounting conservatism on corporate investment during the global financial crisis. Journal of Business Finance \& Accounting, 43(5), 513-542.

Ball, R., \& Shivakumar, L. (2005). Earnings quality in UK private firms: Comparative loss recognition timeliness. Journal of Accounting and Economics, 39(1), 83-128.

Barbosa Filho, F. D. H. (2017). A crise econômica de 2014/2017. Estudos Avançados, 31(89), 5160.

Basu, S. (1997). The conservatism principle and the asymmetric timeliness of earnings. Journal of Accounting and Economics, 24(1), 3-37.

Biddle, G. C., \& Hilary, G. (2006). Accounting quality and firm-level capital investment. The Accounting Review, 81(5), 963-982.

Biddle, G. C., Hilary, G., \& Verdi, R. S. (2009). How does financial reporting quality relate to investment efficiency? Journal of Accounting and Economics, 48(2), 112-131.

Bushman, R. M., Piotroski, J. D., \& Smith, A. J. (2011). Capital allocation and timely accounting recognition of economic losses. Journal of Business Finance \& Accounting, 38(1-2), 1-33.

Campello, M., Graham, J. R., \& Harvey, C. R. (2010). The real effects of financial constraints: Evidence from a financial crisis. Journal of Financial Economics, 97(3), 470-487.

Coelho, A. C. D. (2007). Qualidade informacional e conservadorismo nos resultados contábeis publicados no Brasil. Tese de doutorado não publicada. Universidade de São Paulo, São Paulo, Brasil.

Duchin, R., Ozbas, O., \& Sensoy, B. A. (2010). Costly external finance, corporate investment, and the subprime mortgage credit crisis. Journal of Financial Economics, 97(3), 418-435.

Economatica. (2008). Manual. S. 1.: Autor. Recuperado em 27 maio, 2019, de http://economatica.com/support/manual/portugues/whnjs.htm

Fonseca, P. C. D., Cunha, A. M., \& Bichara, J. D. S. (2013). O Brasil na Era Lula: Retorno ao desenvolvimentismo? Nova Economia, 23(2), 403-428.

Giambiagi, F. (2011). Estabilização, reformas e desequilíbrios macroeconômicos: Os anos FHC. In F. Giambiagi (Org.), Economia brasileira contemporânea: 1945-2010 (2a ed.). Rio de Janeiro, RJ: Elsevier.

Greene, W. (2003). Econometric analysis (5th ed.). New Jersey, United States: Prentice Hall.

Gujarati, D. (2006). Econometria básica. Rio de Janeiro, RJ: Campus-Elsevier. 
Instituto Brasileiro de Geografia e Estatística, Rio de Janeiro. (2016). Contas nacionais trimestrais. Recuperado em 11 dezembro, 2016, de https://servicodados.ibge.gov.br/Download/Download.ashx?http=1\&u=biblioteca.ibge.gov. br/visualizacao/periodicos/2121/cnt_2013_4tri.pdf

Instituto de Pesquisa Econômica Aplicada, Brasília, DF. (2016). Carta de conjuntura. Recuperado em $22 \quad$ novembro, 2016, de http://www.ipea.gov.br/portal/index.php?option=com_content\&view=article\&id=27921\&I temid $=3$

Kammler, E. L., \& Alves, T. W. (2009). Análise da capacidade explicativa do investimento pelo "q" de Tobin em empresas brasileiras de capital aberto. RAE Eletrônica, 8(2), 12.

Kothari, S. P., \& Sloan, R. G. (1992). Information in prices about future earnings: Implications for earnings response coefficients. Journal of Accounting and Economics, 15(2), 143-171.

Lara, J. M. G., Osma, B. G., \& Penalva, F. (2016). Accounting conservatism and firm investment efficiency. Journal of Accounting and Economics, 61(1), 221-238.

Lopes, H. C. (2018). O Brasil no novo milênio: Regulação, progresso técnico e novo desenvolvimentismo. Economia e Sociedade, 27(3), 1029-1052.

Novy, A. (2009). O retorno do Estado desenvolvimentista no Brasil. Indicadores Econômicos FEE, 36(4), 121-128.

Paulani, L. (2017). A experiência brasileira entre 2003 e 2014: Neodesenvolvimentismo? Cadernos do Desenvolvimento, 12(20), 135-155.

Santos, L. M., Costa, D. F., Alberto, J. G. C., Gonçalves, M. A., \& de Faria, E. R. (2011). Análise do $\mathrm{Q}$ de Tobin como determinante do investimento das empresas brasileiras. FACES Journal, 10(3), 65-82. 